\title{
BOUNDED CONVERGENCE OF ANALYTIC FUNCTIONS
}

\author{
BY L. A. RUBEL ${ }^{1}$
}

\begin{abstract}
ABSTRACr. This is a survey of one part of the area of interaction between complex analysis and functional analysis. The space of bounded analytic functions on an open set is considered under numerous topologies, unified by the theme of bounded pointwise convergence. Related problems in approximation theory, the structure of ideals of bounded analytic functions, and inner and outer (also interior and exterior) functions are discussed.
\end{abstract}

I will give a prejudiced survey of some recent developments in the field of interaction between complex analysis and functional analysis. I will also pose a number of unsolved problems. The real measure of the mathematics I will discuss, in my opinion, is the problems it raises and the problems it solves in the hard analysis of bounded analytic functions. The basis of the work at hand is found in my 1966 paper with Shields [23] and in my paper with Ryff [21] that has appeared since this lecture was given. The central theme is the bounded convergence of a sequence of bounded analytic functions.

Let $G$ be an open set in the complex plane, and let $B_{H}(G)$ be the space of all bounded analytic functions on $G$. If $\left\{f_{n}\right\}$ is a sequence of functions in $B_{H}(G)$, we say that $f_{n}$ converges boundedly to $f$, and write $f_{n} \stackrel{B}{\rightarrow} f$, to mean that

(i) $\left\{f_{n}(z)\right\}$ is uniformly bounded in $G$, and

Expanded and improved version of an address delivered before the Madison meeting of the American Mathematical Society on April 18, 1970, by invitation of the Committee to Select Hour Speakers for Western Sectional Meetings.

AMS 1970 subject classifications. Primary 3032, 4602; Secondary 30A24, 30A26, 30A38, 30A40, 30A44, 30A58, 30A76, 30A78, 30A82, 30A98, 31A15, 32A10, 41A10, $41 \mathrm{~A} 20,41 \mathrm{~A} 30,46 \mathrm{~A} 05,46 \mathrm{~A} 20,46 \mathrm{E} 10,46 \mathrm{E} 15,46 \mathrm{E} 25,46 \mathrm{~J} 10,46 \mathrm{~J} 15,46 \mathrm{~J} 20,46 \mathrm{~J} 30$, 54A10, 54A20, 54D55, 54E35.

Key words and phrases. Bounded analytic function, $H^{\infty}$, bounded convergence, bounded weak-star topology, weak-star topology, strict topology, mixed topology, Mackey topology, narrow topology, sequential space, inner function, outer function, interior function, exterior function, universal covering surface, automorphic function, exposed point, extremal function, polynomial approximation, approximation by rational functions, analytic capacity, maximal ideal, complex homomorphism, Gleason part, angular derivative, several complex variables.

1 Research partially supported by a grant from the Air Force Office of Scientific Research, Office of Aerospace Research, United States Air Force, under Grant Number AF OSR 681499. 
(ii) $f_{n}(z) \rightarrow f(z)$ for each $z \in G$.

From the theory of normal families, it follows easily that (ii) may be replaced by

(ii') $f_{n}(z) \rightarrow f(z)$ uniformly on compact subsets of $G$.

Bounded convergence is a natural notion of convergence of a sequence of functions, and we ask whether there is a topology $\tau$ for which $f_{n} \stackrel{r}{\rightarrow} f$ if and only if $f_{n} \stackrel{B}{\rightarrow} f$. The answer is provided by a theorem to be found in [19]. The only thing about bounded convergence to check that is not completely trivial is the following: if it is not the case that $f_{n} \stackrel{B}{\rightarrow} f$, then there is a subsequence $\left\{f_{m}\right\}$ of $\left\{f_{n}\right\}$ such that for every subsequence $\left\{f_{p}\right\}$ of $\left\{f_{m}\right\}$, it is not the case that $f_{p} \stackrel{B}{\rightarrow} f$. This is easy to prove, so there do exist such topologies. In fact there is a strongest such topology, that we designate by $\tau$. In this topology, a set $\Omega$ $\subseteq B_{H}(G)$ is declared open if for each $f \in \Omega$, and each sequence $\left\{f_{n}\right\}$ such that $f_{n} \stackrel{B}{\rightarrow} f$, all but finitely many of the $f_{n}$ must lie in $\Omega$. This is the same as declaring a set $K \subseteq B_{H}(G)$ closed if it is sequentially closed, in the obvious sense.

I am going to conduct an excursion through numerous topologies on $B_{H}(G)$, among them the topology $\tau$ that will appear in several different disguises. Here is a table of the topologies. Don't be dismayed by its length - I will explain them all and many of them will coincide.

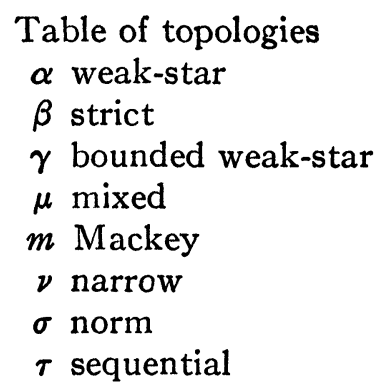

In 1957, Buck [7] introduced the strict topology $\beta$ on $B_{H}(G)$ in the special case where $G=D=\{z:|z|<1\}$ is the unit disc. Shields and I in [23] extensively studied the strict topology in the case of the general region $G$. Let $K(G)$ consist of all nonnegative functions $k$ that are defined and continuous on $G^{-}$, with $k(z)=0$ for all $z \in \partial G$. For $f \in B_{H}(G)$ define the seminorm $\|f\|_{k}$ by

$$
\|f\|_{k}=\sup \{|f(z)| k(z): z \in G\} .
$$

The locally convex topology generated by this family of seminorms is the strict topology $\beta$, and we denote by $\beta(G)$ the space $B_{H}(G)$ under this topology. It is easy to see that $\beta(G)$ is a topological algebra. 
By $H^{\infty}(G)$, I mean $B_{H}(G)$ under the supremum norm $\|f\|_{\infty}$ $=\sup \{|f(z)|: z \in G\}$. The norm topology on $B_{H}(G)$ will be denoted by $\sigma$. In [23] we showed that $H^{\infty}(G)$ is always (isometrically isomorphic to) the dual space of a separable Banach space. Thus, it makes sense to talk of topologies like the weak-star topology $\alpha$. Actually, as early as 1960, Havin found [17], [18] a different representation of $H^{\infty}(G)$ as a dual space, but he did not investigate whether his predual was separable. To consider our duality, let $M(G)$ be the space of bounded complex-valued Borel measures $\mu$ with all their mass in $G$, and subject to the variation norm. Then $M(G)$ is in duality $\left\langle M(G), B_{H}(G)\right\rangle$ with $B_{H}(G)$ via the pairing $\langle\mu, f\rangle$ $=\int f d \mu$. We write $\mu \sim \nu$ to mean that $\int f d \mu=\int f d \nu$ for all $f \in B_{H}(G)$, and let $N(G)=\{\mu: \mu \sim 0\}$. It is easy to see that $N(G)$ is a closed subspace of the Banach space $M(G)$, so that we may form the quotient space

$$
M_{0}(G)=M(G) / N(G) .
$$

We showed, without too much trouble, that

$$
H^{\infty}(G) \cong\left(M_{0}(G)\right)^{*},
$$

where $\cong$ denotes isometric isomorphism. What is not so easy to see is that $M(G) / N(G)$ is separable, since $M(G)$ is certainly not separable. One way we handled this was to prove that for every measure $\mu$ $\in M(G)$, there exists a measure $\nu \in L^{1}(G)$, such that $\nu \sim \mu$ and $\|\nu\|$ $\leqq\|\mu\|$. Here $L^{1}(G)$ consists of those measures in $M(G)$ that are absolutely continuous with respect to planar Lebesgue measure. To sketch our proof, we take first the hardest case, where $\mu$ is a point mass at w-the general case follows from this one by standard balayage formulas from potential theory. Let $\Omega$ be a closed annulus with center at $w$ so chosen that $\Omega \subseteq G$, and let $\nu$ be the Cauchy integral averaged over the radii of the annulus. This means that if the inner radius of $\Omega$ is $a$ and the outer radius is $b$, then for a Borel set $E \subseteq G$,

$$
\nu_{w}(E)=\frac{1}{b-a} \int_{t=a}^{t=b}\left\{\frac{1}{2 \pi i} \int_{|\xi-w|=t} \chi_{E}(\xi) \frac{d \xi}{\xi-w}\right\} d t,
$$

where $\chi_{E}$ is the characteristic function of $E$. In the case of a general measure $\mu \in M(G)$, we let

$$
\nu(E)=\int \nu_{w}(E) d \mu(w) .
$$

It is easy to see that $\nu$ has the required properties. 
It follows that

$$
M(G) / N(G) \cong L^{1}(G) / N^{1}(G),
$$

where $N^{1}(G)=N(G) \cap L^{1}(G)$. Since $L^{1}(G)$ is separable, so is $L^{1}(G) / N^{1}(G)$, and it follows that $M_{0}(G)$ is separable.

On $H^{\infty}(G)$ we may now put the weak-star topology $\alpha$ and the bounded weak-star topology $\gamma$, thinking of $H^{\infty}(G)$ as the dual of $M_{0}(G)$. The topology $\alpha$ is just the topology of convergence on finite subsets of the predual. To describe the topology $\gamma$, let us move to the general context of a Banach space $B$ and its dual $B^{*}$. There are three equivalent formulations of the bounded weak-star topology $\gamma$ on $B^{*}$. First, $\gamma$ is the strongest topology that agrees with $\alpha$ on bounded sets, where $\alpha$ is the weak-star topology. Next, $\gamma$ is the topology of uniform convergence on compact subsets of $B$. Finally, $\boldsymbol{\gamma}$ is the topology of uniform convergence on sequences in $B$ that tend in norm to 0 . One of the main results that Ryff and I proved in [21] is that $\beta=\gamma$. (Since then, Shapiro [27] has placed this result in a general setting. He shows that it holds because the unit ball in $H^{\infty}$ is strictly compact.) Now it is easy to prove that on the dual of a separable Banach space, in the topology $\gamma$, a set is closed if it is sequentially closed. (This should be compared with a result of Banach that in the topology $\alpha$, a convex set is closed if it is sequentially closed.) Hence we have a soft proof of the unpublished result of Paul Hessler that in $\beta(G)$ a subset is closed if it is sequentially closed. From this result, it is easy to prove that $\beta=\tau$, where $\tau$ is the sequential topology introduced earlier. This may be considered a primary reason for studying the topology $\beta$. It emerges as the strongest topology on $B_{H}(G)$ in which a sequence converges if and only if it converges boundedly.

Let me give brief consideration to another topology. Given a vector space with two suitable topologies on it, Wiweger [31] defines the mixed topology $\mu$ that lies, so to speak, halfway between them. In the context of $B_{H}(G), \mu$ lies halfway between the topology of uniform convergence on compact subsets of $G$ and the topology of uniform convergence on all of $G$. Cooper [9] has proved that $\mu=\beta$. This is yet another instance of a metamathematical rule called the "principle of conservation of topologies", to the effect that a natural object like $B_{H}(G)$ cannot support too many different interesting topologies. So far, I have said that $\beta=\tau=\gamma=\mu$. There are several proofs, however, that $\alpha \neq \beta$, and it is trivial that $\sigma \neq \alpha$ and $\sigma \neq \beta$, so that our list of topologies so far reduces to just $\sigma, \alpha, \beta$.

From the abstract characterizations we have iust given of the $\beta$ 
topology, it is now possible to derive many of its properties that Shields and I originally used ad hoc methods for. For example, $\beta$ is complete because the dual of a Banach space, in the bounded weakstar topology, is always complete.

The last topology that I will burden you with here is the Mackey topology $m$ on $H^{\infty}(G)$ as the dual of $M_{0}(G)$. It is the strongest topology on $B_{H}(G)$ that makes it into a locally convex topological vector space whose dual is $M_{0}(G)$. It is also characterized as the topology of uniform convergence on weakly compact, convex, and circled subsets of the predual $M_{0}(G)$.

It is a challenging question, raised by Shields and me in [23], and considered further by Ryff and me in [21] whether $\beta=m$. The evidence is now in that $\beta \neq m$ as soon as $B_{H}(G)$ is not trivial, but to prove it is another matter. This contrasts with the work [28] of my former student B. A. Taylor, who investigated similar topologies on certain spaces of entire functions, and showed that the Mackey topology on them often does coincide with the topology given by a family of suitable weights. That $\beta(G) \neq m(G)$ was shown by Ryff and me to be equivalent to the assertion that there is a weakly compact subset of $M_{0}(G)$ that is not strongly compact. In 1966, Conway [8] proved that $\beta(D) \neq m(D)$, and Ryff and I extended this result to regions $G$ whose boundary contains a free continuum, and it is not hard to prove this (by a second method) for the case where the boundary of $G$ contains any continuum at all. This second method of Ryff and myself proceeds by showing first that $\beta(G) \neq m(G)$ if $G$ supports a nonconstant hypo-inner function. Roughly speaking, a hypo-inner function $f$ on $G$ is an analytic function such that $\|f\|_{\infty} \leqq 1$ and yet $|f(z)|=1$ for $z$ in a set of positive measure on the boundary of $G$.

Let me make this notion precise. Suppose that $G$ supports nonconstant bounded analytic functions, and suppose for convenience that $G$ encloses no removable singularities for all bounded analytic functions. This last means that for each $z_{0} \in \partial G$, there is an $f \in B_{H}(G)$ that does not extend as an analytic function in any neighborhood of $z_{0}$. Then the universal covering surface of $G$ is conformally equivalent to the unit disc $D$ so that we may identify it with $D$. There is a map $\varphi: D \rightarrow G$ that is called the covering map, and the corresponding covering group $\Gamma$ of $G$ is the group of conformal one:one transformations $T$ of $D$ onto itself such that $\varphi(z)=\varphi(T z)$ for all $z \in D$. Every function $f$ in $B_{H}(G)$ lifts via $\varphi^{-1}$ (i.e. $f^{\#}=f \circ \varphi$ ) to a function $f^{\#}$ in $B_{H}(D)$ that is automorphic: $f^{*} \circ T=f^{*}$ for all $T \in \Gamma$.

Now a function $F \in B_{H}(D)$ is called inner if $\|F\|_{\infty} \leqq 1$ and if $\left|F^{*}\left(e^{i \theta}\right)\right|=1$ for almost all $\theta$ (with respect to arc length), where $F^{*}$ 
is the Fatou radial limit function associated with $F ; F^{*}\left(e^{i \theta}\right)$ $=\lim _{r \rightarrow 1} F\left(r e^{i \theta}\right)$, which is defined almost everywhere. Further, $F$ is called hypo-inner in $D$ if only $\|F\|_{\infty} \leqq 1$ and $\left|F\left(e^{i \theta}\right)\right|=1$ for a set of $\theta$ of positive measure. For $f \in B_{I I}(G)$, we say that $f$ is inner or hypoinner, if the lifted function $f^{\prime \prime}$ is inner or hypo-inner, respectively. Ryff and I proved that $\beta \neq m$ in case $G$ supports nonconstant hypoinner functions. It is easy to show that it does in case there is a continuum in $\partial G$. Further, we proved by hammer and tongs classical analysis, that it does in case $G$ is the complement of a closed set $E$ on the real axis, such that $E$ has positive linear measure. In the case of the general region $G$, one would guess that the solution, known to exist, of any of several extremal problems would be inner, and consequently hypo-inner. For example, choose a point $z_{0} \in G$ and consider $f \in B_{H}(G)$ so that $\|f\|_{\infty} \leqq 1$ and $f\left(z_{0}\right)=0$, and maximize $\left|f^{\prime}\left(z_{0}\right)\right|$. The extremal function is the so-called Ahlfors function, and in case $G$ is finitely connected, it is known [2], [3] to be inner.

I conjecture that the hypo-inner functions are just the weak-star exposed points of the unit ball of $H^{\infty}(G)$. Stephen Fisher has shown me in a private communication that this is true in case $G=D$ or if $G$ is, more generally, a finite Riemann surface. If this conjecture were proved, what would be needed next would be a functional-analysis proof that such exposed points exist in abundance, under suitable general hypotheses on the underlying space. I am satisfied that this last topic is a good example of the interworking of the ideas of functional analysis and classical analysis.

Perhaps the main reason for introducing topologies on function spaces is for approximation problems. Let me begin with the problem of polynomial approximation. In 1934, Farrell [12] proved that if $G$ is bounded and connected and if $f$ is a complex function defined on $G$, then there is a sequence $\left\{p_{n}\right\}$ of polynomials such that $p_{n} \stackrel{B}{\rightarrow} f$ if and only if $f$ has a bounded analytic extension to the inside of the outer boundary of $G$. Intuitively, what is meant by the inside of the outer boundary of $G$ is clear. Formally, it is defined as the complement of the closure of the unbounded component of the complement of the closure of $G$. In 1964, Shields and I [22] extended this result to the general bounded open set $G$-it may have infinitely many components. The proof was constructive and used classical methods. Recently, Hoffman (see [29]) has derived a functional analysis proof of Farrell's theorem. His work has been generalized by Ahern and Sarason [1] to the general case considered by Shields and me, with some extensions to bounded approximation by rational functions on some special kinds of sets. Further, Sarason [25], [26] has extended 
Farrell's result from sequential bounded polynomial approximation to polynomial approximation in the weak-star topology $\alpha$. His work had applications in mind to problems of invariant spaces of analytic functions.

Let me interpose here a quick solution to a problem transmitted privately to me by A. Wilansky, related to the work in $[30$, p. 52]. Call a topological space sequential if every sequentially closed set is closed. Improvising terminology, call it super-sequential if the sequential closure of any subset is its topological closure. The question is whether there is a linear topological space that is sequential but not super-sequential. In [4], Arhangel'skil and Franklin showed that there is - their example is actually locally convex. But I can go one step further to give a locally convex topological algebra that is sequential but not super-sequential. It is just $\beta(G)$ where $G$ is the (somewhat pathological) region of Theorem 4.1 of [22]. The subset whose closure is not its sequential closure is just the polynomials, yet we have seen that $\beta(G)$ is a sequential locally convex topological algebra.

Returning to approximation theory, consider the problem of bounded approximation by rational functions. To the best of my knowledge, it is still open, although partial results are known. To be precise, let $G$ be an open set in the complex plane, and let $R_{G}$ be the set of rational functions that belong to $B_{H}(G)$. What is the bounded sequential closure of $R_{G}$ ? When is $R_{G}$ pointwise boundedly (sequentially) dense in $B_{H}(G)$ ?

An interesting related question is the following: let $E$ be a pointwise boundedly dense (p.b.d.) subset of $B_{H}(G)$. Under what further hypotheses must $E$ be strongly pointwise boundedly dense (s.p.b.d.)? Here p.b.d. just means that for each $f \in B_{H}(G)$ there is a sequence $\left\{e_{n}\right\}$ of elements of $E$ such that $e_{n} \stackrel{B}{\rightarrow} f$. And s.p.b.d. means that we may, in addition, require that $\left\|e_{n}\right\|_{\infty} \rightarrow\|f\|_{\infty}$. Now Davie showed in [11] (see also [10]) that if $A(G)$ is p.b.d. then it is s.p.b.d., thus proving a conjecture of Gamelin and Garnett. Here, $A(G)$ is just the space of functions that are continuous on $G^{-}$and analytic on $G$. Further, Gamelin and Garnett have shown in [16] that under suitable special hypotheses on $G, R_{G}$ is s.p.b.d. if it is p.b.d., a problem to which Fisher has contributed in [13]. One might think that if $E$ is a subalgebra of $B_{H}(G)$, then p.b.d. $\Rightarrow$ s.p.b.d., but Davie has privately communicated a counterexample to me.

When is $A(G)$ p.b.d. in $B_{H}(G)$ ? This question has been given an answer by Gamelin and Garnett in [16] in terms of analytic capacity. The analytic capacity $\gamma(K)$ of a compact set $K$ in the complex 
plane is defined by $\gamma(K)=\sup \left|f^{\prime}(\infty)\right|$, where $f$ ranges over all functions defined and analytic on the complement $G$ of $K$ (with respect to the Riemann sphere), with $|f(z)| \leqq 1$ for all $z \in G$ and $f(\infty)=0$. Such an $f$ has an expansion near $\infty$ of the form $f(z)=a_{1} / z+a_{2} / z^{2}+\cdots$ and, by definition, $f^{\prime}(\infty)=a_{1}$. Unfortunately, there is no known way of computing geometrically the analytic capacity of any but the simplest compact sets, although there are some useful inequalities (see, for example the notes [33] of Zalcman) for relating it to computable quantities. To compute $\gamma(K)$ requires knowledge of all bounded analytic functions on $G$. Even the problem of determining geometrical necessary and sufficient conditions that $\gamma(K)=0$ (which is equivalent to $B_{H}(G)$ being trivial), known as the Painleve problem, is still unsolved after some eighty years. (See [20] for a plausible early reference - because of changed standards of rigor, I find it hard to decide whether this specific problem is actually discussed there.)

The notion of s.p.b.d. suggests that it might be profitable to study the narrow topology $\nu$ on $B_{H}(G)$. Here, we follow an idea of Beurling [6] used in harmonic analysis. We say that a sequence $f_{n}$ of functions in $B_{H}(G)$ converges narrowly to $f$, written $f_{n} \stackrel{\nu}{\rightarrow} f$, to mean that

(i) $\left\|f_{n}\right\|_{\infty} \rightarrow\|f\|_{\infty}$ and

(ii) $f_{n}(z) \rightarrow f(z)$ uniformly on compact subsets of $G$.

Then $\nu$ is defined as the strongest topology with this kind of sequential convergence. Now $\nu$ is a metric topology with the metric

$$
\rho(f, g)=\|(f-g) k\|_{\infty}+\left|\|f\|_{\infty}-\|g\|_{\infty}\right|,
$$

where $k$ is any conveniently chosen function that is continuous on $G^{-}$, vanishes on $\partial G$, and vanishes nowhere in $G$. However, the narrow topology is not consistent with the linear structure of $B_{H}(G)$, because 0 plays such a special role-a sequence that converges narrowly to 0 must converge uniformly to 0 . But everybody knows how to approximate 0 by rational functions or whatever!

In another direction, $\beta(G)$ is a topological algebra, so one turns naturally to the study of $\beta$-closed maximal ideals. This is equivalent to considering complex homomorphisms of $B_{H}(G)$ that are $\beta$-continuous. Now even in the case $G=D$, the maximal ideal structure of $H^{\infty}(G)$ is very complicated, and one can hope that things will be simplified by restricting attention to the $\beta$-closed maximal ideals, and this is indeed the case. A complex homomorphism $\Phi$ of $B_{H}(G)$ is $\beta$-continuous precisely when there exists a measure $\mu \in M(G)$ such that $\Phi(f)=\int f d \mu$ for all $f \in B_{H}(G)$. In this case, we say that $\Phi$ is a distinguished homomorphism. A first question is how much a distinguished homomorphism must resemble evaluation at a point of $G$. 
In [23], Shields and I showed that if the boundary of $G$ is a union of continua, then the distinguished homomorphisms are just the point evaluations, $\Phi(f)=f\left(z_{0}\right)$ where, $z_{0}$ ranges over $G$. In [32], Zalcman gave a simple proof that for the general region $G$, the distinguished homomorphisms must all belong to the same Gleason part as the point evaluations. (To say that two complex homomorphisms $\Phi$ and $\Psi$ belong to the same Gleason part is to say that $\|\Phi-\Psi\|<2$, where the norm is the linear functional norm.) It follows from the work of Behrens [5] that this Gleason part may contain complex homomorphisms that are not distinguished. In [15], Gamelin and Garnett prove that any Gleason part that contains a distinguished homomorphism contains a homeomorphic image of $\beta(N) \backslash N$, where $\beta(N)$ is the Stone-Cech compactification of the discrete space $N$ of positive integers. Hence, any Gleason part contains a non-distinguished homomorphism. Rudin [24] gave an example of an infinitely connected plane domain with a distinguished homomorphism that is not point evaluation (including evaluation at removable singularities for bounded analytic functions). Gamelin and Garnett showed [15] that there is at most one distinguished homomorphism $\Phi$ in the fiber over a point $z_{0} \in G^{-}$. To say that $\Phi$ lies in this fiber is to say that $\Phi(f)$ $=f\left(z_{0}\right)$ for all $f \in B_{H}(G)$ that are analytic at $z_{0}$. In particular, $\Phi(p)$ $=p\left(z_{0}\right)$ for every polynomial $p$ if $G$ happens to be bounded. Every homomorphism lies in some fiber. It would be interesting to characterize geometrically those points in the boundary of $G$ that have a distinguished homomorphism in the fiber over them. This has been done in terms of analytic capacity by Gamelin and Garnett in [15], but I have already expressed my reservations about analytic capacity results in the present stage of development. In the same paper, they prove a result (Theorem 3.5) that implies the $\beta$ corona conjecture that the point evaluations are dense in the space of distinguished homomorphisms.

Let me now make a connection with, and generalization of, some classical function theory. In [23], Shields and I called a function $f \in B_{H}(G)$ exterior when the principal ideal generated by $f$ is dense in $\beta(G)$. If $G=D$, then the exterior functions are just the outer functions, i.e., those functions $f$ such that

$$
f(z)=\exp \frac{1}{2 \pi} \int_{-\pi}^{\pi} \frac{z+e^{i \theta}}{z-e^{i \theta}} d \mu(\theta)
$$

where $\mu$ is absolutely continuous with respect to Lebesgue measure. It is an important problem to give a geometrical characterization of 
the exterior functions on the general open set $G$. In particular, one would expect that if $f$ is a nonvanishing function in $B_{H}(G)$, then $f$ being exterior or not is a local property of $f$ on the boundary. In his forthcoming thesis, my student $\mathrm{C}$. W. Kennel proves some surprising results in this direction, even in the case $G=D$. First of all, it is now clear that if $f$ is exterior in $G$ and if $G^{\prime} \subseteq G$, then $f^{\prime}=f \mid{ }_{G^{\prime}}$ is exterior in $G^{\prime}$. Also, if $G$ is simply connected, and if $\varphi: D \rightarrow G$ is a Riemann map of $D$ one: one onto $G$, then $f \circ \varphi$ is outer in $D$. Kennel proves that given any nonvanishing $f \in B_{H}(D)$, we may construct two simply connected regions $G_{1}$ and $G_{2}$ in $D$, with $G_{1} \cup G_{2}=D$, so that $f_{1}=f \mid G_{1}$ and $f_{2}=\left.f\right|_{c_{3}}$ are both exterior functions. The question of how being exterior depends on the domain is a delicate one. Kennel proves the following result in this direction. In $D$, the function, defined for $z \in D$,

$$
a(z)=\exp \left(\frac{z+1}{z-1}\right)
$$

is sometimes called the "world's best function" and sometimes called the "world's worst function". I propose here the name, the "atomic function". It is very far from being an outer function. At any rate, let $G$ be a simply connected subset of $D$ and let $a^{\prime}=\left.a\right|_{a}$. For certain domains $G, a^{\prime}$ is an exterior function, and for others, it is not. Those domains for which it is not are characterized by Kennel as follows. Let $\varphi: D \rightarrow G$ be a Riemann map, and look at those points $e^{i t} \in \partial D$ for which $\varphi^{*}\left(e^{i t}\right)=1$, where $\varphi^{*}$ is the Fatou radial limit function associated with $\varphi$. The condition is that there should exist at least one such point at which $\varphi$ has an angular derivative. This means that $\lim \left\{(\varphi(z)-1) /\left(z-e^{i t}\right)\right\}$ should exist as $z \rightarrow e^{i t}$ nontangentially. This means roughly that the boundary of $G$ is tangential to the boundary of $D$ at the point 1. For example, it follows from this result that if $G$ is the unit disc with the nonnegative real axis removed, then $\exp \{(z+1) /(z-1)\}$ is exterior on $G$, a somewhat surprising fact.

The characterization by Gamelin and Garnett referred to above, of those points $z_{0}$ in the boundary of $G$ that have a distinguished homomorphism in the fiber over them, is in terms of the convergence of a so-called Melnikov series. Kennel proves, when $G$ is bounded, and encloses no removable singularities for $B_{H}(G)$, that the divergence of this series is necessary and sufficient that the function $z-z_{0}$ be exterior. Thus, under these mild conditions on $G, z_{0}$ has no distinguished homomorphism lying over it if and only if $z-z_{0}$ is exterior. Gamelin and Garnett have since privately communicated a direct proof of this fact. 
In [23], Shields and I also defined an interior function $f$ as one for which the principal ideal $(f)$ generated by $f$ is $\beta$-closed. In $D$, the interior functions are just the products of inner functions by units. (A unit is a function $f \in B_{H}(G)$ such that $1 / f \in B_{H}(G)$. This just says that $f$ should be bounded away from 0 in $G$.) My student, C. W. Neville, is now investigating the in terior-exterior factorization on general regions $G$, and also for which regions $G$ the result holds that Shields and I proved for $D$, namely that every $\beta$-closed ideal is principal. He has found some geometrical conditions on $G$ that are sufficient for an analogous result to hold, and has shown that these conditions cannot be completely dropped, but his results are not yet complete.

Finally, one can pose the analogous questions about interior and exterior functions for domains in complex $n$ space. Since this lecture was given, Shields and I have proved that the function $f\left(z_{1}, z_{2}\right)$ $=z_{1}$ has no interior-exterior factorization in the unit ball $B^{2}$ $=\left\{\left(z_{1}, z_{2}\right):\left|z_{1}\right|^{2}+\left|z_{2}\right|^{2}<1\right\}$ and that the function $g\left(z_{1}, z_{2}\right)=z_{1}-z_{2}$ has no interior-exterior factorization in the polydisc $D^{2}$ $=\left\{\left(z_{1}, z_{2}\right):\left|z_{1}\right|<1,\left|z_{2}\right|<1\right\}$.

In summary, I hope I have given some feeling for the interworking of functional analysis and classical analysis in one area of mathematics. Nothing would please me more than to have stimulated some interest in this area, both by the results I have described, and by the problems I have posed.

\section{REFERENCES}

1. P. R. Ahern and D. Sarason, On some hypo-Dirichlet algebras of analytic functions, Amer. J. Math. 89 (1967), 932-941. MR 36 \#4338.

2. L. V. Ahlfors, Bounded analytic functions, Duke Math. J. 14 (1947), 1-11. MR $9,24$.

3. - Open Riemann surfaces and extremal problems on compact subregions, Comment. Math. Helv. 24 (1950), 100-134. MR 12, 90; MR 13, 1138.

4. A. V. Arhangel'skir and S. P. Franklin, Ordinal invariants for topological spaces, Michigan Math. J. 15 (1968), 313-320, 506. MR 39 \#2112.

5. M. F. Behrens, The maximal ideal space of algebras of bounded analytic functions on infinitely connected domains, Thesis, U.C.L.A., 1969.

6. A. Beurling, Un thêorème sur les fonctions bornées et uniformément continues sur l'axe réel, Acta Math. 77 (1945), 127-136. MR 7, 61.

7. R. C. Buck, Algebraic properties of classes of analytic functions, Seminars on Analytic Functions, vol. II, Princeton University, Princeton, N. J., 1957, pp. 175-188.

8. J. B. Conway, The strict topology and compactness in the space of measures. I, Bull. Amer. Math. Soc. 72 (1966), 75-78. MR 32 \#4509.

9. J. B. Cooper, The strict topology and spaces with mixed topologies (preprint).

10. A. M. Davie, Bounded approximation and Dirichlet algebras, Summer Gathering on Function Algebras (Aarhus, 1969), Various Publ. Series, no. 9, Matematisk Institut, Aarhus Universitet, Aarhus. 
11. - Bounded approximation and Dirichlet sets (preprint).

12. O. J. Farrell, On approximation by polynomials to a function analytic in a simply connected region, Bull. Amer. Math. Soc. 41 (1934), 707-711.

13. S. D. Fisher, Bounded approximation by rational functions, Pacific J. Math. 28 (1969), 319-326. MR 39 \#1663.

14. - On Schwarz's lemma and inner functions, Trans. Amer. Math. Soc. 138 (1969), 229-240. MR 39 \#1651.

15. T. W. Gamelin and J. Garnett, Distinguished homomorphisms and fiber algebras (preprint).

16. - Pointwise bounded approximation and Dirichlet algebras (preprint).

17. V. P. Havin, On the space of bounded regular functions, Dokl. Akad. Nauk SSSR 131 (1960), 40-43 Soviet Math. Dokl. 1 (1960), 202-204. MR 22 \#11277.

18. - On the space of bounded regular functions, Sibirsk. Mat. Ž. 2 (1961), 622-638; English transl., Amer. Math. Soc. Transl. (2) 53 (1966), 202-220. MR 25 \#2425.

19. J. Kisyński, Convergence du type $\mathscr{L}$, Colloq. Math. 7 (1959-60), 205-211. MR 23 \#A615.

20. P. Painlevé, Sur les lignes singulières des fonctions analytiques, Ann. Fac. Sci. Univ. Toulouse 2 (1888).

21. L. A. Rubel and J. V. Ryff, The bounded weak-star topology and the bounded analytic functions, J. Functional Analysis 5 (1970), 167-183.

22. L. A. Rubel and A. L. Shields, Bounded approximation by polynomials, Acta Math. 112 (1964), 145-162. MR $30 \# 5104$.

23. - The space of bounded analytic functions on a region, Ann. Inst. Fourier (Grenoble) 16 (1966), fasc. 1, 235-277. MR 33 \#6440.

24. W. Rudin, Essential boundary points, Bull. Amer. Math. Soc. 70 (1964), 321324. MR 28 \#3167.

25. D. Sarason, On the order of a simply connected domain, Michigan Math. J. 15 (1968), 129-133. MR 37 \#3326.

26. - Weak-star generators of $H^{\infty}$, Pacific J. Math. 17 (1966), 519-528. MR $35 \# 2151$.

27. J. H. Shapiro, Weak topologies on subspaces of $C(S)$ (preprint).

28. B. A. Taylor, Some locally convex spaces of entire functions, Proc. Sympos. Pure Math., vol. XI, Amer. Math. Soc., Providence, R. I., 1968, pp. 431-467.

29. J. Wermer, Seminar über Funktionen-Algebren, Lecture Notes in Math., no. 1, Springer-Verlag, Berlin, 1964. MR $35 \# 5947$.

30. A. Wilansky, Topics in functional analysis, Lecture Notes in Math., no. 45, Springer-Verlag, Berlin and New York, 1967. MR 36 \#6901.

31. A. Wiweger, Linear spaces with mixed topology, Studia Math. 20 (1961), 47-68. MR 24 \#A3490.

32. L. Zalcman, Bounded analytic functions on domains of infinite connectivity, Trans. Amer. Math. Soc. 144 (1969), 241-269.

33. - Analytic capacity and rational approximation, Lecture Notes in Math., no. 50, Springer-Verlag, Berlin and New York, 1968. MR 37 \#3018.

UNIVERSITY OF IlLINOIS, URBANA, IlLINOIS 61801 